\title{
Is a test of cure necessary following treatment for cervical infection with Chlamydia trachomatis?
}

\author{
K W Radcliffe, D Rowen, D E Mercey, G Mumtaz, G L Ridgway, A J Robinson, J S Bingham
}

\begin{abstract}
Tests of cure (TOC) were performed on specimens from 106 women following treatment for chlamydial infection of the cervix. 91 women attended for the first TOC with enzyme immunoassay (EIA) within one week of finishing antibiotics. Three were EIA positive, 88 were EIA negative. These three women were subsequently EIA and culture negative although they received no further antibiotics. Ninety women returned for the second TOC with EIA and culture between seven and 27 days after completing treatment. All the results were negative. Routine TOC is unnecessary following appropriate antichlamydial therapy. If TOC is indicated antigen-detection methods should not be used immediately after finishing antibiotics as misleading positive results may be obtained. EIA or culture at one week or later after treatment is reliable.
\end{abstract}

Introduction

Infection of the lower genital tract with Chlamydia trachomatis is one of the commonest sexually transmitted diseases. It is common clinical practice following treatment to repeat microbiological tests to ensure chlamydiae have been eradicated. This study was undertaken to investigate whether such a test of cure (TOC) is necessary and if so whether enzyme immunoassay (EIA) or cell-culture is the more appropriate test and how long after completion of treatment it should be done.

\section{Patients and methods}

Female patients attending the departments of Genitourinary Medicine at the Middlesex and University College Hospitals who had positive cervical cultures for C. trachomatis were recruited into the study.

Departments of Genitourinary Medicine

K W Radcliffe, D Rowen, D E Mercey, A J Robinson, J S Bingham

Department of Medical Microbiology, University College and Middlesex Hospitals, London. G Mumtaz, A J Robinson
Suitable patients were identified in two ways:

(1) Women attending the departments were screened for chlamydiae by EIA (Chlamydiazyme, Abbott Laboratories). Women with positive EIA had cultures taken prior to treatment;

(2) Women attending as contacts of men with nongonococcal urethritis (NGU) had cultures taken before receiving antibiotics.

Informed consent was obtained from all subjects. Treatment prescribed was either doxycycline $100 \mathrm{mg}$ twice a day or oxytetracycline $500 \mathrm{mg}$ four times a day, for seven days.

Subjects were advised to abstain from intercourse until cure was established. They were asked to return the day after finishing antibiotics for TOC with EIA and after a further seven days for a second TOC with EIA and culture. Women attending more than seven days after completing treatment were excluded from analysis of the first TOC.

Specimens for EIA were collected, transported and examined according to the manufacturer's recommendations. Positive results were confirmed by a specific blocking antibody test. Specimens giving equivocal results were further examined by direct immunofluorescence (Microtrak, Syva Company) of the EIA buffer as described in a previous study. ${ }^{1}$

Specimens for culture were obtained from the endocervical canal using cotton-wool tipped swabs after the ectocervix had been wiped with a cottonwool ball. Specimens were transported to the laboratory in 2-sucrose phosphate medium and inoculated onto duplicate monolayers of cycloheximide-treated McCoy cells. After incubation for $\mathbf{4 8}$ to 72 hours one culture was fixed, stained with iodine and examined for typical inclusions. If no chlamydiae were detected the second culture was passaged onto fresh McCoy cells and after a further $\mathbf{4 8}$ to $\mathbf{7 2}$ hours incubation was examined by direct immunofluorescence (Syva Microtrak C. trachomatis Culture Confirmation Test).

\section{Results}

One hundred and six women returned for one or both TOC. Culture was positive prior to treatment in 105. One was culture-negative but positive to both EIA 
and direct immunofluorescence (DFA) and was therefore included.

Fifty six women were treated with doxycycline and 49 with oxytetracycline. One woman was pregnant and received erythromycin $500 \mathrm{mg}$ twice a day for seven days.

Ninty one women attended for the first TOC. One returned one day prior to completing treatment, the rest between one and seven days after finishing therapy. EIA was negative in 88 , three were EIA positive. These three women were all examined the day after finishing antibiotics and all were EIA and culture negative at their second TOC seven, eight and fourteen days later respectively, having received no further treatment. Seventy five of these women also attended for the second TOC and all were both EIA and culture negative.

A total of 90 women returned for the second TOC between seven and 27 days after treatment. All 90 were culture negative. Eighty nine were also EIA negative (EIA was omitted in one).

\section{Discussion}

In this study microbiological cure was established in all 90 women who returned for the final TOC. This is consistent with the following laboratory and clinical evidence which indicates that antichlamydial therapy is extremely effective unless there is non-compliance or the patient is reinfected.

In vitro assays show $C$. trachomatis to be highly susceptible to the tetracyclines and erythromycin. ${ }^{2}$ Naturally occurring resistance of chlamydiae to these antibiotics has never been described. ${ }^{3}$

In the largest series of women followed after treatment for cervical infection with C. trachomatis, 161 women were examined repeatedly for up to 20 months after taking oxytetracycline $250 \mathrm{mg}$ four times a day for 21 days. ${ }^{4}$ Chlamydiae were detected by culture in $16(10 \%)$ at times between two weeks and 12 months. However, ten of these women had current partners with proven gonorrhoea, NGU or chlamydial infection, and the remaining six were all having intercourse with the original or a new partner. Therefore, reinfection and not treatment failure was the probable explanation for the re-isolation of chlamydiae in all cases.

Erythromycin has been shown to be as effective as oxytetracycline in the treatment of cervical infection with $C$. trachomatis. ${ }^{5}$

The results of this present study indicate that routine TOC following therapy for cervical chlamydial infection is unnecessary. This accords with the advice of both the Centers for Disease Control $^{6}$ and the World Health Organisation. ${ }^{7}$

Clinical follow-up will still be necessary in the majority of cases: to assess compliance, to exclude possible reinfection, to exclude adverse effects of antibiotics and for further contact tracing. The physician may feel that a TOC is indicated in cases where non-compliance or reinfection is suspected.

If a TOC is required an antigen-detection method should not be used immediately after finishing treatment. Both EIA and DFA can give misleading positive results in this situation owing to the detection of persisting antigens from non-viable chlamydiae. ${ }^{8}$ This is the probable explanation of the positive EIA results in the three women $(3.3 \%)$ in this study who were subsequently EIA and culture negative. In a similar study comparing EIA and culture as TOC one subject ( $4 \%)$ was EIA positive but culture negative on the seventh (and final) day of therapy with erythromycin but was EIA and culture negative one week later. ' Another study by Nachamkin et al evaluated DFA as TOC and found that $7 / 38(18 \%)$ patients gave borderline results seven to ten days after the completion of treatment. ${ }^{10}$

Patients who have just completed a course of antichlamydial therapy will have a low prevalence of C. trachomatis. The results of antigen-detection tests have lower positive predictive values in lowprevalence populations ${ }^{8}$ and less confidence could be placed in a positive result in such circumstances.

Use of cell-culture immediately after cessation of treatment would also be inadvisable as sufficient antibiotic may be present to inhibit growth of chlamydiae.

The results of this study indicate that EIA or culture is reliable for TOC when performed one week or later after finishing therapy. This is consistent with the results of Lefebvre et al who found $100 \%$ correlation between EIA, culture and DFA when used for TOC four weeks or longer after treatment. ${ }^{11}$ Further studies are necessary to elucidate whether DFA is suitable for TOC performed earlier than four weeks.

In summary, TOC is not necessary following appropriate antichlamydial therapy unless noncompliance or reinfection is suspected. If the clinician believes TOC is indicated EIA or culture should be taken one week or later after finishing antibiotics.

Address for correspondence: Dr K W Radcliffe, Department of Genitourinary Medicine, Charing Cross Hospital, London W6 8RF, UK.

1 Mumtaz G, Ridgway GL, Nayagam A, et al. Enzyme immunoassay compared with cell culture and immunofluorescence for detecting genital chlamydia. J Clin Pathol 1989;42:658-60.

2 Johannisson G. Studies on Chlamydia trachomatis as a cause of lower urogenital tract infection. Acta Dermatol 1981;supp 93:1-55.

3 Mourad A, Sweet RL, Sugg N, et al. Relative resistance to erythromycin in Chlamydia trachomatis. Antimicrob Agents Chemother 1980;18:696-8. 
4 Rees A, Tait A, Hobson D, et al. Chlamydia in relation to cervical infection and pelvic inflammatory disease. In: Hobson $\mathrm{D}$, Holmes $\mathrm{KK}$ (ed). Non-gonococcal urethritis and related infections. Washington, American Society for Microbiology, 1977.

5 Oriel JD, Ridgway GL. Comparison of erythromycin and oxytetracycline in the treatment of cervical infection by Chlamydia trachomatis. J Infect 1980;2:259-62.

6 Centers for Disease Control, 1989 sexually transmitted diseases treatment guidelines. $M M W R$ 1989;38 (S-8):1-43.

7 STD treatment strategies. WHO consultation on development of sexually transmitted disease treatment strategies 1989. Geneva WHO/VDT/98.447.

$8 \mathrm{Kellogg}$ JA. Clinical and laboratory considerations of culture vs antigen assays for detection of Chlamydia trachomatis from genital specimens. Arch Pathol Lab Med 1989;113:453-60.

9 Havlicheck DH, Mauck C, Mummaw NL et al. Comparison of chlamydial culture with Chlamydiazyme assay during erythromycin PCE treatment of chlamydia genital infections. Sex Transm Dis 1990;17:48-50.

10 Nachamkin I, Sawyer K, Skalina D, et al. Test-of-cure analysis by direct immunofluorescence for Chlamydia trachomatis after antimicrobial therapy. J Clin Microbiol 1987;25:1774-5.

11 Lefebvre J, Lapperriere $\mathrm{H}$, Rousseau $\mathrm{H}$, et al. Comparison of three techniques for detection of Chlamydia trachomatis in endocervical specimens from asymptomatic women. J Clin Microbiol 1988;26:726-31.

Accepted for publication 17 September 1990 . 